\title{
Treated Hypertension has No Influence on the Efficacy of Alendronate in the Therapy of Postmenopausal Osteoporosis: A Non-Randomized, Non- Blind, Controlled Prospective Study
}

\author{
Doerte Matziolis ${ }^{*}, 1$, Monika Drewke ${ }^{2}$, Georg Matziolis ${ }^{1}$ and Carsten Perka ${ }^{1}$

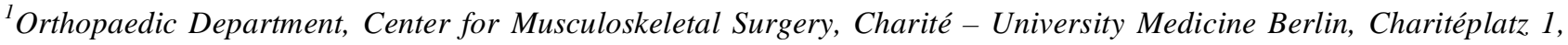 \\ D-10117 Berlin, Germany \\ ${ }^{2}$ General Medical Practice, Lindenstr. 39, D-15848 Friedland/Beeskow, Germany
}

\begin{abstract}
Changes in hormone metabolism during menopause are involved in the development of osteoporosis and arterial hypertension. A mutual influence of these two clinical pictures is evident on the basis of pathophysiological factors.

In this study, we investigated whether a drug therapy of hypertension influences the efficacy of a bisphosphonate (alendronate) in the treatment of postmenopausal osteoporosis. For this purpose, 60 female patients were enrolled in the study, 30 of them on drug treatment for hypertension in addition to requiring treatment for osteoporosis, while the control group was only suffering from osteoporosis.

Bone density was measured at the start of the study and 6 months after therapy (QCT).

A significant increase in bone density was demonstrated in both groups, while a difference between the groups could not be seen.

Treated arterial hypertension does not have a negative influence on the treatment of postmenopausal osteoporosis with alendronate. Rather, the concomitant treatment of these two clinical pictures would appear to make sense, in order to reduce morbidity and mortality.
\end{abstract}

Keywords: Osteoporosis, hypertension, alendronate, bisphosphonate.

\section{INTRODUCTION}

The menopause is a risk factor in the development of cardiovascular diseases and osteoporosis [1]. Hormonal changes as part of the aging process in women, with a reduction in oestrogen and progesterone, are the cause [2]. Hormone replacement therapy would therefore appear to make sense for prevention. Combination prophylaxis with oestrogen and progesterone led to an increase in bone density and a reduction of the fracture risk [3], but did not show a reduction in the incidence of myocardial infarction and tripled the risk of thrombosis (HERS-II study) [4]. Further studies showed an increase in the risk of breast cancer (WHI study) [2], so that, despite being a causative approach, the prophylaxis of postmenopausal cardiovascular diseases and osteoporosis with hormone replacement is no longer a standard therapy. Instead, the independent symptomatic therapy of osteoporosis and metabolic syndrome (hypertension, hypercholesterolaemia and hyperglycaemia) is recommended, since untreated hypertension is the main cause of cardiovascular events [5, 6].

*Address correspondence to this author at the Center for Musculoskeletal Surgery, Charité - University Medicine Berlin, Charitéplatz 1, D-10117 Berlin, Germany; Tel: +49 30450652 206; Fax: +49 30450515 922;

E-mail: doerte.matziolis@charite.de
On the basis of the data available and the pathophysiological factors, a mutual influence of hypertension and osteoporosis is evident. The occurrence of pathological fractures that accompanies reduced bone density is associated with cardiovascular diseases [7]. An imbalance in calcium metabolism plays a role in the pathophysiology of hypertension [8,9]. Since bone is the largest calcium store in the human body, the bone mineral salt content can be changed pathologically by the release of calcium from the bone. Osteoporosis could thus be induced by untreated hypertension $[7,10]$. This clinical observation is additionally supported by the findings of Bastos et al. on spontaneous hypertensive rats that presented a reduced bone quality and elevated bone resorption markers [11]. On the other hand it recently has been shown, that Beta-blockers are independently associated with fragility fractures [12]. In contrast Olmos et al. recently showed that bone mineral density was increased by thiazides in hypertensive postmenopausal women [13]. Up to now, it has been unclear whether antihypertensive therapy influences the efficacy of drug therapy for osteoporosis. The objective of this study was, therefore, to elucidate whether female patients treated with antihypertensives who are suffering from postmenopausal osteoporosis benefit to the same extent from treatment with a bisphosphonate as female patients without hypertension. 


\section{METHODS}

Sixty female patients suffering from postmenopausal osteoporosis were enrolled in this monocentric, prospective, non-blind, controlled study. This study was conducted in an orthopaedic practice after written approval from the ethics committee. To begin with, the files were inspected for patient selection. If there was anamnestic or clinical evidence of osteoporosis, a bone density measurement was taken by means of QCT. The DVO-REKO osteoporosis risk questionnaire was completed by all female patients who came into question. This questionnaire covers the following risk factors for osteoporosis: reduction in height, acute backache, increased tendency to fall over, peripheral fractures after bagatelle trauma, relatives with osteoporosis, medical history of femoral head fractures, intake of glucocorticoids, presence of a chronic inflammatory intestinal disease, reduction of physical activity, underweight, calcium intake $<1000 \mathrm{mg} / \mathrm{d}$, fish $<2 \mathrm{x} /$ week, menopause before the age of 45 years).

If the diagnosis of postmenopausal osteoporosis was confirmed by a bone density of less than $80 \mathrm{mg} / \mathrm{ml}$ calcium hydroxylapatite [14] and at least one positive response in the DVO-REKO osteoporosis risk questionnaire, the patients were enrolled in the study after giving their consent. The patients were subsequently allocated to the study groups depending on whether or not they were receiving drug therapy for hypertension and the kind of medication was documented. Systolic and diastolic blood pressure were measured in all patients before and after therapy. Exclusion criteria were the presence of secondary osteoporosis, the regular intake of glucocorticoids, chronic inflammatory diseases of the gastrointestinal tract, renal dysfunction, unstable arterial hypertension, secondary hypertension, condition after myocardial infarction or stroke, known hypocalcaemia or osteomalacia.

All patients enrolled received basic therapy of their osteoporosis with alendronate as directed, with additional substitution of calcium and vitamin D (DVO guideline 2006).

Bone density was determined again by means of QCT 6 months after inclusion in the study.

A visual analogue scale (VAS) was used to measure pain intensity, and the IOF-41-Qualeffo score at baseline and at the 6-month follow up was recorded to determine quality of life [15]. The latter consists of a total of 41 questions that cover the different aspects that account for quality of life (pain, mobility, leisure time, perception of health, mood). The score is standardised to a percentage result by means of the following formula:

(current score - 41) x 100

\section{4}

A high percentage value indicates a strong burden caused by osteoporosis.

A power analysis was performed and based on a bone mineral density difference of $10 \mathrm{mg} / \mathrm{ml}$ that is considered to be clinically relevant and should therefore be detected in the intergroup comparison. The expected BMD of osteoporosis patients was set to $50 \pm 15 \mathrm{mg} / \mathrm{ml}$ resulting from empirical data of the authors last years of clinical practice. Defining a level of significance of 0.05 and a power of $80 \%$, this resulted in a required sample size of 30 patients per group.

\section{RESULTS}

Sixty female patients were enrolled in this study. The mean age in the control group was 67.3 years ( 49 - 80 years) and in the hypertension group 71.2 years (58 - 82 years) $[\mathrm{p}=$ 0.021]. In the control group, the mean systolic blood pressure was $122(100-140) \mathrm{mmHg}$ and the mean diastolic value $78(70-90) \mathrm{mmHg}$ before therapy. Likewise in the hypertension group, as a result of the drug therapy, the mean systolic value was $135(120-160) \mathrm{mmHg}$ and the mean diastolic value $81(75-90) \mathrm{mmHg}$ before the start of the study. After therapy, the mean systolic blood pressure in the hypertension group was $133(115-170) \mathrm{mmHg}$ and the mean diastolic value $79(70-90) \mathrm{mmHg}$. This corresponds to upper normal values according to the guidelines of the Deutsche Hochdruckliga (German Hypertension League). The kind of antihypertensive medication is given in Table $\mathbf{1 .}$

Table 1. Baseline Characteristics of the Study Collective

\begin{tabular}{|c|c|c|c|}
\hline & Hypertension & Control & p-Value \\
\hline \hline Age [years] & $71.2(58-82)$ & $67.3(49-80)$ & 0.021 \\
\hline $\begin{array}{c}\text { Systolic Blood } \\
\text { Pressure } \\
{[\mathrm{mmHg}]}\end{array}$ & $135(120-160)$ & $122(100-140)$ & n.s \\
\hline $\begin{array}{c}\text { Diastolic Blood } \\
\text { Pressure [mmHg] }\end{array}$ & $81(75-90)$ & $78(70-90)$ & n.s. \\
\hline $\begin{array}{c}\text { Bone Mineral } \\
\text { Density [mg/dl] }\end{array}$ & $56.2(37.0-79.3)$ & $50.0(22.2-75.9)$ & n.s. \\
\hline
\end{tabular}

In the control group, the mean systolic values were 121 $(100-135) \mathrm{mmHg}$ and the mean diastolic values 78 (70 80) $\mathrm{mmHg}$. This corresponds to normal blood pressure values. The systolic blood pressure values did not differ significantly between the two groups before and after alendronate therapy (Fig. 1).

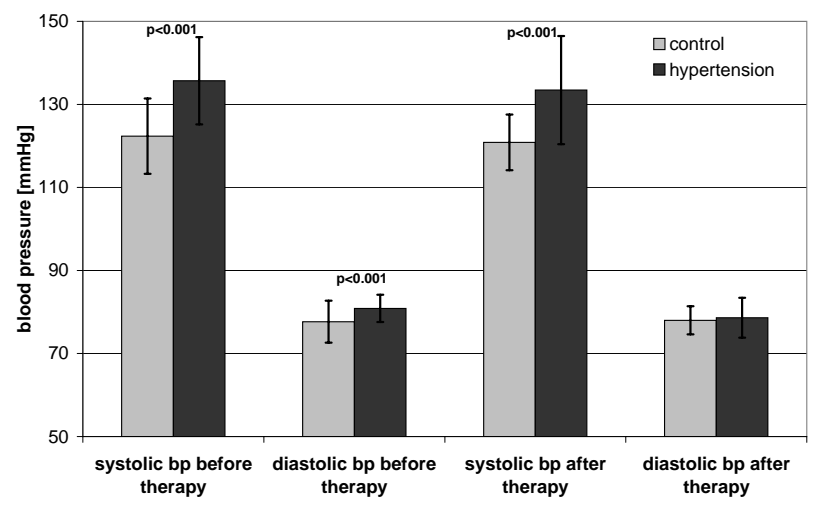

Fig. (1). Systolic and diastolic blood pressure before and after alendronate therapy.

Bone density in the hypertension group was $56.2 \mathrm{mg} / \mathrm{dl}$ $(37.0-79.3 \mathrm{mg} / \mathrm{dl})$ at the start of the study and increased within 6 months over the course of therapy with alendronate to $59.9 \mathrm{mg} / \mathrm{dl}(32-82 \mathrm{mg} / \mathrm{dl})[\mathrm{p}=0.002]$. In the control 
group, bone density was $50.0 \mathrm{mg} / \mathrm{dl}(22.2-75.9 \mathrm{mg} / \mathrm{dl})$ before therapy and increased to $55.9 \mathrm{mg} / \mathrm{dl}(30-81.9 \mathrm{mg} /$ dl) ( $<$ 0.001; Fig. 2). However, if one compares the hypertension group and the control group, significant differences in bone density are not to be found, either at the start of the study or at follow up.

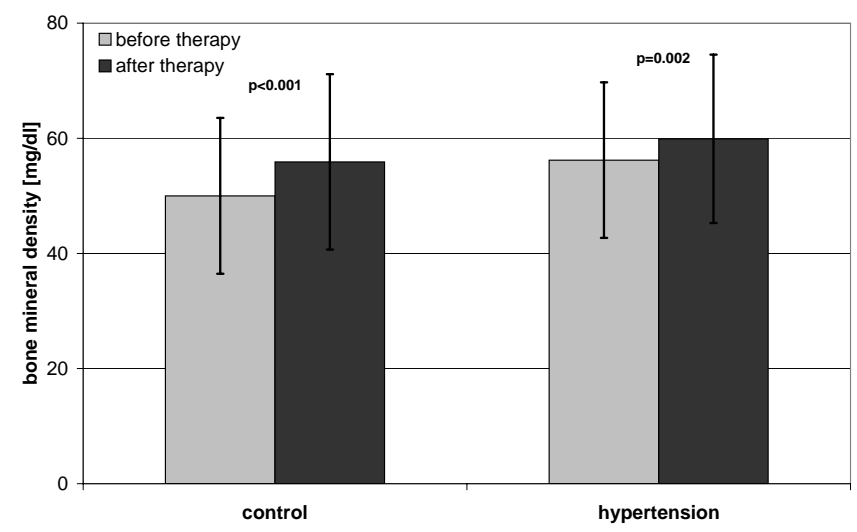

Fig. (2). Bone density before and after alendronate therapy

In the therapy of osteoporosis-associated pain, again a significant improvement could be shown in both groups over the course of the study. The VAS decreased over the observation period from $55 \mathrm{~mm}$ to $41 \mathrm{~mm}$ [p $<0.001]$ in the control group and from $61 \mathrm{~mm}$ to $42 \mathrm{~mm}(\mathrm{p}<0.001$; Fig. 3) in the hypertension group. The IOF-41-Qualeffo has a domain that reflects the subjects' subjective pain perception. Here, the patients in the control group showed a reduction of $53(5-85)$ to $46(5-80)$ points. In the hypertension group, the values changed from $48(0-90)$ to $39(0-80)$ points [p < 0.001]. However, no significant differences could be found between the two groups before and after therapy in the IOF41-Qualeffo/ pain or in the VAS.

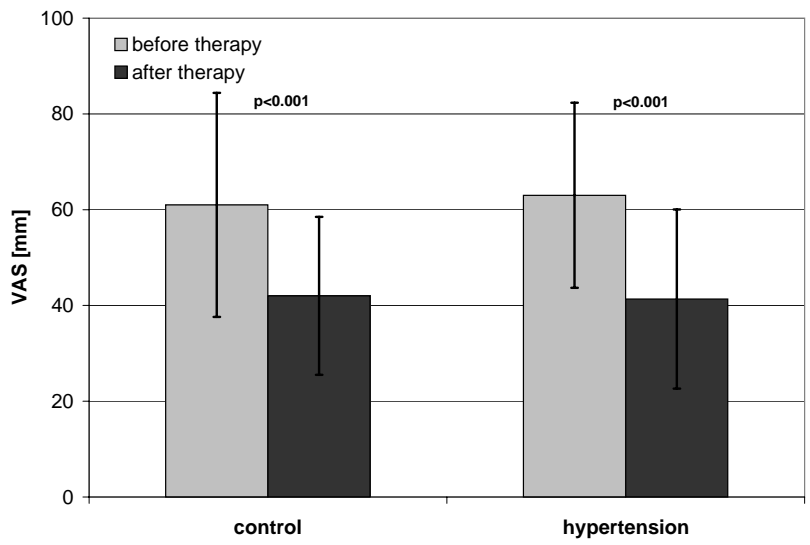

Fig. (3). Visual analogue scale before and after alendronate therapy.

If one compares the other domains of the IOF-41Qualeffo and their total before and after therapy separately for the two treatment groups, an improvement in quality of life in all individual domains and in their total as a result of the alendronate therapy could be demonstrated for the hypertension group. There were also significant improvements in the individual domains in the control group, with the exception of the "mood" domain (Fig. 4).

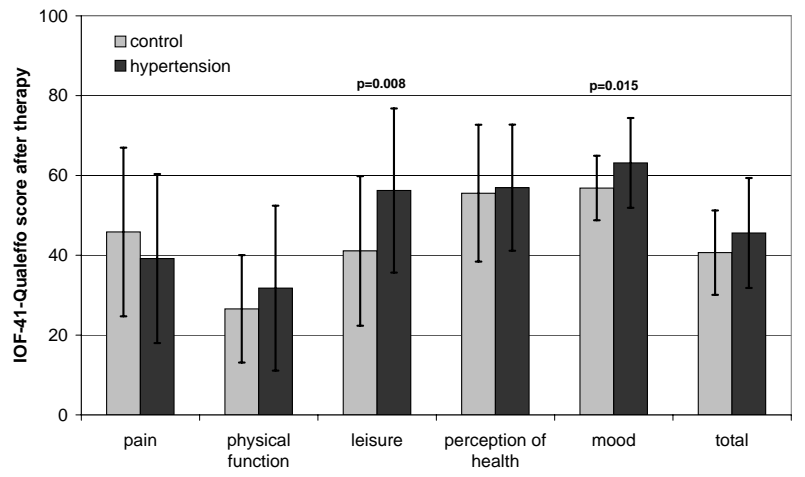

Fig. (4). IOF-41-Qualeffo score 6 months after alendronate therapy.

If one compares the results of the hypertension and control group, again no differences are to be found in the IOF-41-Qualeffo. Here, the hypertension group already showed significantly higher values in the sub-domains leisure time and mood before therapy, compared with the control group [ $p=0.005$ and $p=0.025]$. These group differences were not affected by the therapy $(p=0.008$ and $\mathrm{p}=0.015$; Fig. 4).

Taking the patients age and the sub-domains leisure time and mood of the IOF-41-Qualeffo as confounding variables into account all group comparisons were re-calculated but did not result in any new statistically significant difference in terms of blood pressure, BMD, VAS, IOF-41-Qualeffo. The kind of antihypertensive medication did not statistically significantly influence any of the examined parameters.

\section{DISCUSSION}

The results presented here show that a drug treatment of arterial hypertension does not have any negative influence on the treatment of postmenopausal osteoporosis with alendronate. Clinical studies have demonstrated that there is a connection between cardiovascular diseases and an increased risk of fracture. Thus, Schulz et al. showed that aortal calcification is accompanied by a 3 -fold higher risk of femoral head fracture [16]. In the reverse case, Tanko et al. showed that osteoporosis patients have a 4-fold higher risk of suffering a stroke or myocardial infarction than patients with osteopaenia [1]. Other working groups have shown that there is a connection between cardiovascular diseases and the loss of bone mass $[17,18]$.

A therapy of osteoporosis and hypertension would therefore appear to be essential in order to reduce the risk of cardiovascular as well as osteoporotic morbidity and mortality $[1,19,20]$.

There is widespread evidence of a mutual interaction of the two clinical pictures [7-10].

There is evidence of a molecular biological origin of the coincidence of osteoporosis and hypertension. The two diseases are linked via the RANKL/RANK/osteoprotegerin system. Osteoprotegerin is a glycoprotein that belongs to the group of the tumour necrosis factor receptor superfamily and can prevent a differentiation of the precursor cells of osteoclasts and thus osteoporosis [21, 22]. Reduced osteoprotegerin leads to a reduction in bone density and can lead to arteriosclerosis [23]. 
The hormone oestrogen, which is reduced in postmenopausal osteoporosis, can lead to a reduction in bone density and have an influence on atherogenesis via the release of cytokines (IL-1, IL-6 and TNF-alpha) and also via a reduction of osteoprotegerin [24].

The study presented here collected purely clinical data and did not investigate calcium metabolism or determine osteoprotegerin levels.

In our study group, the patients of the hypertension group did not have reduced bone density compared with the control group, either in the baseline situation or after therapy with alendronate.

In accordance with the literature, we were able to show that bone density already increased after 6 months' initial therapy with a bisphosphonate [25]. The presence of arterial hypertension did not have any influence on the therapeutic outcome.

A reduction in pain and an improvement in quality of life could be shown in both groups over the follow-up period. This is consistent with the results of Dursun et al., who also showed a reduction in osteoporosis-associated pain and an improvement in quality of life under therapy with a bisphosphonate in the treatment of postmenopausal osteoporosis [26]. The presence of arterial hypertension did not have an influence on the pain reduction or the improvement in quality of life after alendronate therapy.

Limitations of the study are the non-randomized, nonblind design and the limited number of cases. Possibly confounding variables that showed differences between the groups before therapy were the patients' age and subdomains of the IOF-41-Qualeffo score resulting from the nonrandomized study design. This was statistically addressed by a re-calculation taking these possible confounders into account but did not alter the conclusions.

The follow-up period was long enough to detect therapeutical effects of both, antihypertensive and osteoporosis therapy but it remains unclear whether antihypertensive therapy may influence the efficacy of alendronate in the long term (Table 2).

Table 2. Antihypertensive Therapy in the Hypertension Group (16 Patients had a Monotherapy, 10 Patients Received a Double Combination, Three Patients a Triple Combination, and Two Patients a Quadruple Combination of Antihypertensive Drugs)

\begin{tabular}{|c|c|}
\hline Antihypertensive Medication & Patients \\
\hline \hline Beta-Blocker & 18 \\
\hline Calcium Antagonist & 10 \\
\hline ACE Inhibitor & 9 \\
\hline Diuretic & 5 \\
\hline Nitrate & 5 \\
\hline Angiotensin II Antagonist & 3 \\
\hline
\end{tabular}

Although fracture incidence is a clinically more relevant outcome parameter for a successful osteoporosis therapy the change of bone mineral density was chosen in this study based on the fact that it shows quantitative effects on small patient collectives and is more robust against confounding variables (e.g. risk of falling, neurological disorders).

Based on the results, hypertension under drug therapy does not have a negative influence on a 6 month treatment of postmenopausal osteoporosis with alendronate. Rather, a good drug therapy of these two diseases would appear to make sense, in order to reduce their morbidity and mortality.

\section{REFERENCES}

[1] Tanko LB, Christiansen C, Cox DA, et al. Relationship between osteoporosis and cardiovascular disease in postmenopausal women. J Bone Miner Res 2005; 20: 1912-20.

[2] Rossouw JE, Anderson GL, Prentice RL, et al. Risks and benefits of estrogen plus progestin in healthy postmenopausal women: principal results from the Women's Health Initiative randomized controlled trial. JAMA 2002; 288: 321-33.

[3] Cauley JA, Robbins J, Chen Z, et al. Effects of estrogen plus progestin on risk of fracture and bone mineral density: the Women's Health Initiative randomized trial. JAMA 2003; 290: 1729-38.

[4] Grady D, Herrington D, Bittner V, et al. Cardiovascular disease outcomes during 6.8 years of hormone therapy: Heart and Estrogen/progestin Replacement Study follow-up (HERS II). JAMA 2002; 288: 49-57.

[5] Vitale C, Miceli M, Rosano GM. Gender-specific characteristics of atherosclerosis in menopausal women: risk factors, clinical course and strategies for prevention. Climacteric 2007; 10(Suppl 2): 1620.

[6] Rosano GM, Vitale C, Tulli A. Managing cardiovascular risk in menopausal women. Climacteric 2006; 9(Suppl 1): 19-27.

[7] Cappuccio FP, Meilahn E, Zmuda JM, et al. High blood pressure and bone-mineral loss in elderly white women: a prospective study. Study of Osteoporotic Fractures Research Group. Lancet 1999; 354: 971-5.

[8] Power ML, Heaney RP, Kalkwarf HJ, et al. The role of calcium in health and disease. Am J Obstet Gynecol 1999; 181: 1560-9.

[9] Zittermann A, Schleithoff SS, Koerfer R. Putting cardiovascular disease and vitamin D insufficiency into perspective. $\mathrm{Br} \mathrm{J}$ Nutr 2005; 94: 483-92.

[10] von der Recke P, Hansen MA, Hassager C. The association between low bone mass at the menopause and cardiovascular mortality. Am J Med 1999; 106: 273-8.

[11] Bastos MF, Brilhante FV, Gonçalves TE, et al. Hypertension may affect tooth-supporting alveolar bone quality: a study in rats. J Periodontol 2010; 81(7): 1075-83.

[12] Sosa M, Saavedra P, Mosquera J, et al., GIUMO Cooperative Group. Beta-blocker use is associated with fragility fractures in postmenopausal women with coronary heart disease. Aging Clin Exp Res 2010; [Epub ahead of print].

[13] Olmos JM, Hernández JL, Martínez J, et al. Bone turnover markers and bone mineral density in hypertensive postmenopausal women on treatment. Maturitas 2010; 65(4): 396-402.

[14] Banzer D, Felsenberg D. Computerized tomography determination of bone mineral content in quantitative diagnosis of osteoporosis. Orthopade 1989; 18(1): 12-7.

[15] Lips P, Cooper C, Agnusdei D, et al. Working Party for Quality of Life of the European Foundation for Osteoporosis. Quality of life in patients with vertebral osteoporosis. Validation of the quality of life questionnaire of the European Foundation for Osteoporosis (Qualeffo). Osteoporosis Int 1999; 10: 150-60.

[16] Schulz E, Arfai K, Liu X, et al. Aortic calcification and the risk of osteoporosis and fractures. J Clin Endocrinol Metab 2004; 89: 4246-53.

[17] Pennisi P, Signorelli SS, Riccobene S, et al. Low bone density and abnormal bone turnover in patients with atherosclerosis of peripheral vessels. Osteoporos Int 2004; 15: 389-95.

[18] Kiel DP, Kauppila LI, Cupples LA, et al. Bone loss and the progression of abdominal aortic calcification over a 25 year period: the Framingham Heart Study. Calcif Tissue Int 2001; 68: 271-6. 
[19] Marcovitz PA, Tran HH, Franklin BA, et al. Usefulness of bone mineral density to predict significant coronary artery disease. Am J Cardiol 2005; 96: 1059-63.

[20] Bevilacqua M, Dominguez LJ, Rosini S, et al. Bisphosphonates and atherosclerosis: why? Lupus 2005; 14 : 773-9.

[21] Schoppet M, Preissner KT, Hofbauer LC. RANK ligand and osteoprotegerin: paracrine regulators of bone metabolism and vascular function. Arterioscler Thromb Vasc Biol 2002; 22: 54953

[22] Collin-Osdoby P. Regulation of vascular calcification by osteoclast regulatory factors RANKL and osteoprotegerin. Circ Res 2004; 95: $1046-57$
[23] Bucay N, Sarosi I, Dunstan CR, et al. Osteoprotegerin-deficient mice develop early onset osteoporosis and arterial calcification. Genes Dev 1998; 12: 1260-8.

[24] McFarlane SI. Bone metabolism and the cardiometabolic syndrome: pathophysiologic insights. J Cardiometab Syndr 2006; 1: 53-7.

[25] Hosking D, Chilvers CE, Christiansen C, et al. Prevention of bone loss with alendronate in postmenopausal women under 60 years of age. Early Postmenopausal Intervention Cohort Study Group. N Engl J Med 1998; 338: 485-92.

[26] Dursun N, Dursun E, Yalcin S. Comparison of alendronate, calcitonin and calcium treatments in postmenopausal osteoporosis. Int J Clin Pract 2001; 55: 505-9.

(C) Matziolis et al.; Licensee Bentham Open.

This is an open access article licensed under the terms of the Creative Commons Attribution Non-Commercial License (http://creativecommons.org/licenses/by$\mathrm{nc} / 3.0 /$ ) which permits unrestricted, non-commercial use, distribution and reproduction in any medium, provided the work is properly cited. 